\title{
Effect of Liposome Curcumin Combined with PDGF-BB on Tendon Healing in Rats
}

Wang TL" ${ }^{1 \#, ~ W u ~ W J ~}{ }^{1 \#, ~ G u ~ J ~ X ~}{ }^{1}$ and Hou XR ${ }^{2 *}$

${ }^{1}$ Department of Hand and Foot Surgery, Yangzhou University, China

${ }^{2}$ Department of Surgery, Soochow University, China "Equally Contributed

*Corresponding author: Hou XR, Department of Surgery, Soochow University, RuiHua Affiliated Hospital, Road No5 Tayun, Suzhou 215104, Jiangsu, China

Received: February 17, 2021; Accepted: March 13, 2021; Published: March 20, 2021

\begin{abstract}
Objective: To study the effect of liposomal curcumin combined with PDGFBB on tendon healing after tendon injury in rats and explore its mechanism. Methods Thirty-six rats were randomly divided into four groups: healthy control group (group A), liposomal curcumin group (group B), PDGF-BB group (group C) and combined application group (group D). The rats in each group were divided into groups according to the experimental group. At the $4^{\text {th }}$ and $8^{\text {th }}$ weeks after operation, the general morphology, histomorphology, biomechanics and other aspects of rat tendon were detected and statistically analyzed.
\end{abstract}

Results: The degree of tendon adhesion in group $B$ and $D$ was significantly less than that in group $\mathrm{A}$ and $\mathrm{C}$, and the difference was statistically significant. The biomechanical test showed that the tendons in group $C$ and $D$ had higher strength and could withstand greater stress than those in group $A$ and $B$.

Conclusion: Liposome curcumin combined with PDGF-BB can significantly reduce the degree of adhesion after tendon injury, increase the strength of tendon healing, and significantly promote tendon healing.

Keywords: Curcumin; Liposomes; Cytokines; Tendon healing; Tendon adhesion; Animal experiments

\section{Introduction}

Because of the multiple tendon injuries and the difficulty of repairing tendon adhesion after tendon injury has always been a problem. Difficulties to be solved urgently in clinical work. According to the current research results, the healing mechanism of tendon can be divided into two ways: endogenous healing and exogenous healing. Endogenous healing is the process of tendon healing by stimulating synovial fluid and cytokines. Tendon cells and tendon stem cells in tendon can proliferate, differentiate and migrate. Exogenous healing is achieved by the proliferation of fibroblasts in peritendinous tissue and the formation of granulation tissue along with the capillary growing into the tendon section. From the mechanism analysis, endogenous healing can improve the biomechanical properties of tendon tissue. Exogenous healing can not only repair tendons, but also increase the formation of tendon adhesion, thus affecting tendon sliding. Curcumin has the functions of lowering blood lipid, antitumor, anti-inflammatory, choleretic and anti-oxidation. In acute and chronic inflammation, curcumin can play a very good protective role in vivo [1]. The purpose of this experiment is to study the effect and effect of liposome curcumin on inhibiting tendon adhesion and promoting tendon healing, and to explore and analyze its mechanism.

\section{Materials and Methods}

\section{Materials and Instruments}

Preparation of liposome curcumin: $100 \mathrm{mg}$ PLGA was dissolved in $2 \mathrm{~mL}$ DCM containing curcumin for $15 \mathrm{~min}$. After PLGA was completely dissolved, $200 \mathrm{~L} \mathrm{H} 2 \mathrm{O}$ (W1) was added to PLGA solution $(\mathrm{O})$, and the mixture was ultrasonic shocked $3 \mathrm{~min}$ to form water in oil emulsion (W1/O). The W1/O emulsion was added to the $10 \mathrm{~mL}$
5\% (w/w) PVA solution, and the ultrafine grinding 3 min was carried out by using the Scientz-IID probe supersonic apparatus at once. The mixture was added to $50 \mathrm{~mL} 0.3 \%$ (w/w) PVA solution (W2) to form a multiple emulsion (W1/O/W2). The W1/O/W2 emulsion was stirred overnight to evaporate the remaining DCM. PLGA nanoparticles were collected by centrifugation, washed with deionized water three times, and then lyophilized into powder.

Establishment and grouping of tendon injury model: Fortyeight rats aged 5-6 months were selected, weighing about 350-400 grams (all rats were purchased from the Animal Laboratory Center of Jiangsu University). The experimental animals were anaesthetized by intraperitoneal injection of $5 \%$ chloral hydrate and fixed prone on the operating table. Routine skin preparation and disinfection, "S" shape incision of bilateral Achilles tendon skin, can be seen Achilles tendon divided into thick and thin two strands. Two strands of tendons were separated bluntly, and then the thicker Achilles tendon was cut with a sharp blade at the transition point of proximal tendon about $3 \mathrm{~mm}$, and the Achilles tendon insertion point about $2 \mathrm{~mm}$. The core suture was made with Prolene 5-0 line and two groups of modified "Kessler" method. Line 1-0 sutures the skin incision intermittently, disinfects again, and fixes it firmly in an extended position. According to different experimental groups, different drug treatments were injected into the incision. The experiment has been approved by relevant departments. (Laboratory animal license: SYXK(SU)2017-0044, Department of science and technology, JiangSu, China)

Forty-eight rats were randomly divided into four groups with 12 rats in each group. The specific grouping is as follows: Healthy control group (group A): $2 \mathrm{ml}$ saline; Liposome curcumin group (group B): 1 $\mathrm{ml}$ liposome curcumin $(1 \mathrm{mg} / \mathrm{L})+1 \mathrm{ml}$ saline; PDGF-BB group (group 
C): $1 \mathrm{ml}$ 50ug/LPDGF-BB+1 ml saline; Combined application group (group D): $1 \mathrm{ml}$ liposome curcumin $+1 \mathrm{ml}$ 50ug/LPDGF-BB

After the operation, the external fixation and braking of lower limbs were maintained. Drugs were injected in different groups every 3 days. In this experiment, 6 rats were selected at each time point at the 4 th and 8 th week after operation. The $3 \mathrm{~mm}$ tendons on both sides of bilateral tendon segments were removed for examination.

\section{Detection methods}

Gross tendon adhesion score: Adhesion tissues on the palm and back of tendon were examined. Peritendinous adhesion was divided into the following types: I. Non-adhesion. There was no adhesion around the tendon, but there was a small amount of granulation tissue. II. Film-like adhesion. There were only a few membranous adhesions, which had no effect on tendon sliding. III. loose adhesion. Slender, loose, soft fibers and tendons indicate easy separation. IV. Medium dense adhesion. Medium texture, tendon mobility; V. Dense adhesion. Hard, often limited, but poorly mobile, deep into the tendon parenchyma, and muscles. Tendons are not segmented obviously and are not easy to be segregated.

Histochemical staining: After general observation, tendon sheaths and tendon tissues within $2 \mathrm{~mm}$ on both sides of anastomotic stoma were taken and routinely embedded in paraffin. Longitudinal sections of tendons were made, slices were 5 microns thick, and HE staining was performed routinely. Cell proliferation, migration and collagen fibrillation in tendon repair site were observed by $\mathrm{HE}$ staining. The specific contents of adhesion under microscope are shown in Table 1 .

Biomechanical measurement: The rats were selected $f$ or biomechanical test at the 8 th week after operation. After the animals were killed with carbon dioxide, the prone position was fixed on the operating table. The knee joint of the hind limb was severed sharply in $S$ shape. The peritendinous tissue around the tendon anastomosis was separated and removed with tissue scissors. The distal part of the free Achilles tendon and the foot were fixed at the lower end of the mechanical tester, and the proximal part of the Achilles tendon of the rat was fixed at the upper end of the mechanical tester. The preload is $20 \mathrm{~g}$ in weight. Zero the tester before mechanical testing. Tendon sliding distance: Tendon sliding distance was measured when the maximum pulling force was $50 \mathrm{~N}$ and the stretching speed was $1 \mathrm{~cm} /$ min. Maximum Tensile Strength: Measure the Tensile Strength (N) required when the tendon is pulled off. The above results are directly generated by computer.

\section{Statistical analysis}

Statistical analysis was used to analyze the statistical differences among the groups. Kruskal Wallis test and Nemenyi method were used to analyze the difference of tendon adhesion score among different groups. The biomechanical results of different groups, including the Table 1: Microscopic adhesion classification.

\begin{tabular}{|l|l|c|}
\hline \multicolumn{1}{|c|}{ Number of fibers } & \multicolumn{1}{c|}{ Fiber quality } & Score \\
\hline No significant adhesions & No significant adhesions & 0 points \\
\hline A small amount of fibers & Regularly and longer & 1 point \\
\hline Medium fibers & Irregular and uneven length & 2 points \\
\hline Lots of fibers & Dense, non-separable & 3 points \\
\hline
\end{tabular}

Table 2: Statistical test of tendon adhesion score.

\begin{tabular}{|c|c|c|c|c|c|c|c|c|}
\hline & & $\begin{array}{l}\text { Intergroup } \\
\text { comparison" }\end{array}$ & A\&B & $A \& C$ & $A \& D$ & $B \& C$ & B\&D & C\&D \\
\hline \multirow{2}{*}{$4^{\text {th }}$ weeks } & $x^{2}$ & 17.048 & 27.65 & 0.24 & 11.33 & 33.07 & 3.58 & 14.88 \\
\hline & $\mathrm{P}^{*}$ & 0.001 & 0 & 0.89 & 0 & 0 & 0.17 & 0 \\
\hline \multirow{2}{*}{$8^{\text {th }}$ weeks } & $x^{2}$ & 15.126 & 28.61 & 1.89 & 9.99 & 45.22 & 4.79 & 20.58 \\
\hline & $\mathrm{P}^{*}$ & 0.002 & 0 & 0.39 & 0.01 & 0 & 0.09 & 0 \\
\hline
\end{tabular}

Note: "Kruskal-wallis test ${ }^{*} \mathrm{P}<0.05$, the difference was statistically significant.

maximum stress and maximum displacement, were analyzed by oneway ANOVA (one-way ANOVA analysis), and multiple comparisons were made by LSD. The above tests were statistically analyzed by SPSS software (Chicago, USA, version 22.0).

\section{Results}

\section{Gross morphological observation}

The gross tendon score of each group was shown in Figure 1. There were different degrees of adhesion in each group 4 weeks after operation. Group A and C were the most important, while group B and $\mathrm{D}$ were lighter. Compared with 4 weeks after operation, the degree of tendon adhesion in each group was relatively reduced at 8 weeks after operation. Kruskal-Wallis test was used for statistical analysis, and Nemenyi test was used for statistical analysis among groups. The statistical results are shown in Table 2. There was significant difference in the gross tendon adhesion score of each group $(\mathrm{P}=0.001$ at week 4 and $\mathrm{P}=0.002$ at week 8 ). Further analysis of tendon adhesion scores among groups showed that there was significant difference between group $\mathrm{A}$ and $\mathrm{C}$, group $\mathrm{B}$ and group $\mathrm{D}$, while there was no significant difference between group A and group C, group B and group D.

\section{Histomorphological observation}

Microscopic grading of tendon adhesion was performed in each group, and the results were shown in Figure 2. Among them, the degree of adherence in group $\mathrm{C}$ was higher than that in other groups; the degree of adherence in group B was relatively lighter than that in group D. Statistical analysis was performed by Kruskal-Wallis test (as shown in Table 3). At week 4, there was no significant difference in the scores of tendon adhesion between groups $(\mathrm{P}=0.2)$; at week 8 , there was significant difference in the scores of tendon adhesion between groups $(\mathrm{P}=0.14)$. Further analysis of the endoscopic adhesion score (Nemenyi test) at the 8th week showed that there was no significant difference among the groups.

\section{Biomechanical testing}

At the 8th week, biomechanical tests were carried out for each

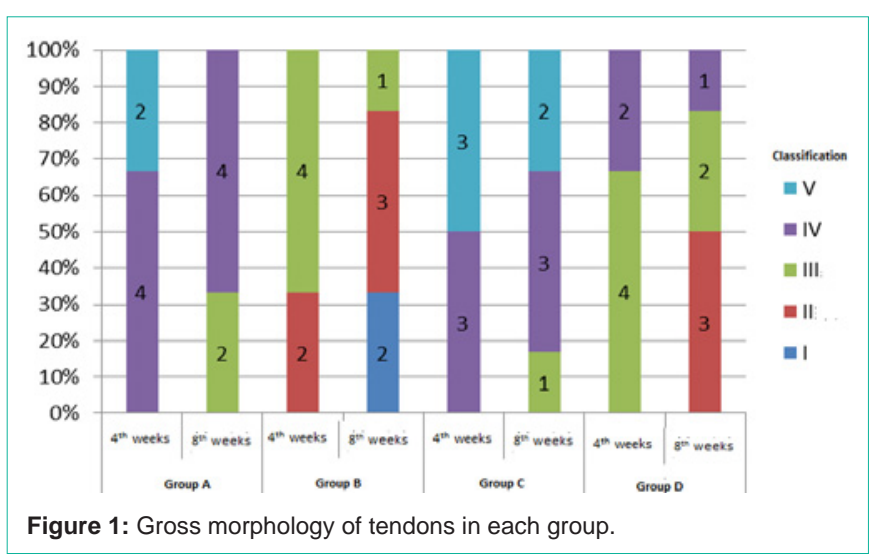




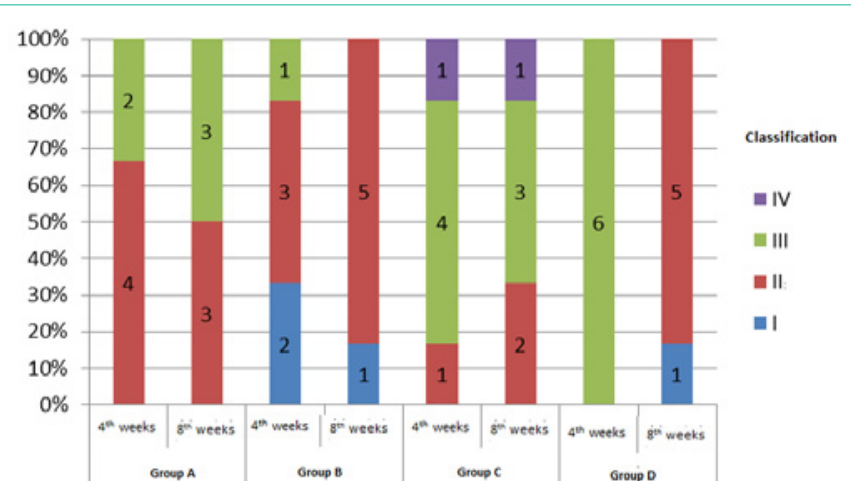

Figure 2: Morphological observation of adhesion grading under tendon endoscopy in each group.

Table 3: Statistical test of tendon adhesion score under microscope.

\begin{tabular}{|c|c|c|}
\hline \multirow{2}{*}{$4^{\text {th }}$ weeks } & $\mathrm{X}^{2}$ & 9.889 \\
\cline { 2 - 3 } & $\mathrm{P}^{*}$ & 0.2 \\
\hline \multirow{2}{*}{$8^{\text {th }}$ weeks } & $\mathrm{X}^{2}$ & 10.617 \\
\cline { 2 - 3 } & $\mathrm{P}^{*}$ & 0.014 \\
\hline
\end{tabular}

Note: ${ }^{\mathrm{P}}<0.05$, the difference was statistically significant

Table 4: Biomechanical test results of each group (Mean \pm SD).

\begin{tabular}{|c|c|c|}
\hline & Maximum Tensile Force (N) & Maximum Displacement (mm) \\
\hline Group A: & $29.97 \pm 4.24$ & $11.58 \pm 1.31$ \\
\hline Group B: & $23.73 \pm 5.53$ & $10.91 \pm 2.68$ \\
\hline Group C: & $39.14 \pm 3.76$ & $9.70 \pm 1.50$ \\
\hline Group D: & $36.66 \pm 10.48$ & $12.93 \pm 0.89$ \\
\hline Total & $32.37 \pm 8.67$ & $11.28 \pm 2.01$ \\
\hline$P^{*}:$ & 0.03 & 0.3 \\
\hline
\end{tabular}

Note: ${ }^{*} \mathrm{P}<0.05$, the difference was statistically significant.

Table 5: Statistical analysis of maximum pulling force between groups.

\begin{tabular}{|c|c|c|c|c|c|c|}
\hline group & A\&B & A\&C & A\&D & B\&C & B\&D & C\&D \\
\hline$P^{*}:$ & 0.116 & 0.025 & 0.093 & 0.001 & 0.003 & 0.521 \\
\hline
\end{tabular}

Note: ${ }^{*} \mathrm{P}<0.05$, the difference was statistically significant.

group of samples, and the results were shown in Figures 3-6. For the analysis of the results (Table 4 \&Table 5), the maximum tendon tension of group A and group B was significantly less than that of group $\mathrm{C}$ and group $\mathrm{D}$. The results of single factor ANOVA analysis showed that the difference was statistically significant. There was no significant difference in the maximum end displacement between the samples.

\section{Discussion}

Curcumin is a yellow powder extracted from the rhizome of Curcuma longa, which is a Dione compound. Some medical studies have shown that curcumin has the effects of lowering blood lipid, anti-tumor, anti-inflammatory, choleretic and anti-oxidation. Curcumin can play a very good protective role in acute and chronic inflammation [1]. Curcumin can reduce the aggregation of key inflammatory chemokines and related cytokines (IL-6, IL-10, IFN, etc.) in tissues, and can significantly reduce the inflammatory response in tissues. This conclusion has been confirmed in a variety of animal experimental models [2,3]. However, current studies have shown that curcumin is almost insoluble in water, easily oxidized, poorly

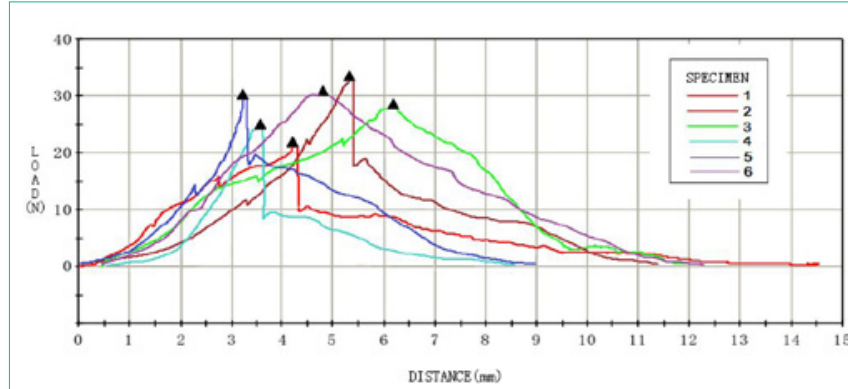

Figure 3: Biomechanical test results of normal control group (group A).

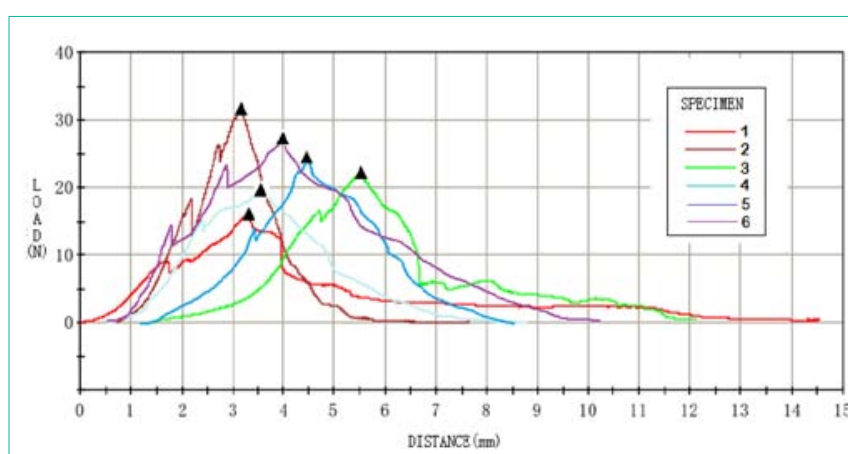

Figure 4: Biomechanical test results of liposome curcumin group (group B).

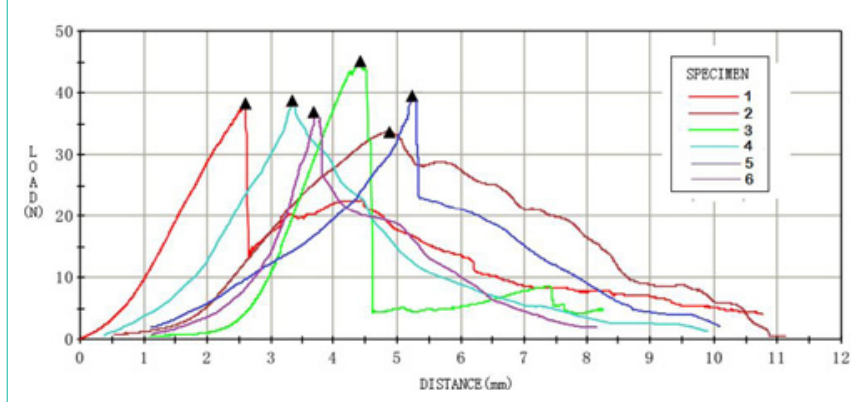

Figure 5: Biomechanical test results of PDGF BB group (group C).

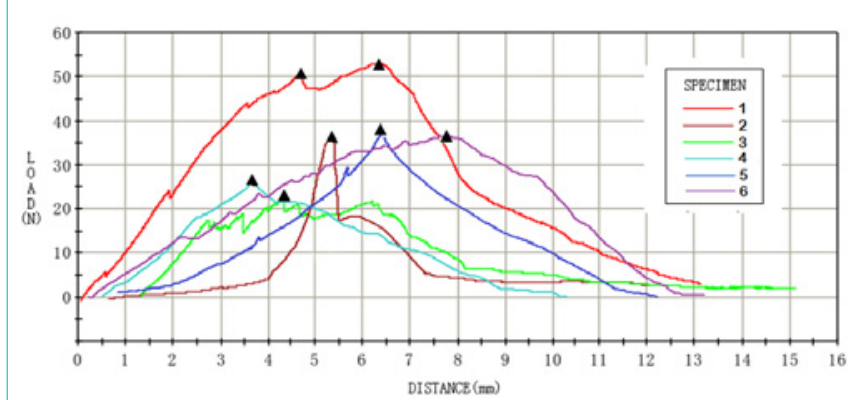

Figure 6: Biomechanical test results of combined application group (group D).

absorbed in vivo and has low bioavailability. Therefore, the application of curcumin in vivo experiments must be carried through a carrier to improve the bioavailability and thus play its pharmacological role. Liposome, as a kind of drug carrier with early clinical application and most mature development, has good targeting and sustained-release effect $[4,5]$. For Curcuma longa, many experimental papers have confirmed that it has the shortcomings of improving the insolubility 
of natural curcumin in water and poor bioavailability, and has achieved remarkable results in related experiments [6]. Experiments have proved that curcumin can significantly reduce the occurrence of tendon adhesion in the model of tendon injury. This may be due to its ability to alleviate inflammation in tendons and peritendinous tissues and to weaken the exogenous healing of tendons. PDGF is the mitogen and chemokine of all kinds of cells, especially fibroblasts. It has three isomers, PDGF-AA, PDGF-AB and PDGF-BB. PDGF$\mathrm{BB}$ has a significant effect on wound healing. Current experiments show that [7-9], in order to improve the content of cytokine PDGF$\mathrm{BB}$ in tendon injury tissue, it is helpful to improve the strength and toughness of tendon tissue after healing. In normal tissues, only a lower concentration of PDGF-BB is produced. Its main function is chemotaxis to neutrophils, mononuclear macrophages and fibroblasts $[10,11]$. Thus, more fibroblasts accumulate at the broken ends of tendons and promote the proliferation of blood vessels. While promoting the exogenous healing of tendons, the adhesion was aggravated to a certain extent. The purpose of this experiment is to promote the growth of tendon fibers and accelerate the healing of tendon tissue by increasing the local concentration of PDGF-BB. However, after combined application of liposome curcumin, the degree of tendon adhesion was significantly reduced compared with PDGF-BB alone.

To sum up, curcumin is encapsulated in liposome granules, which solves the problem of insoluble in water and has the possibility of local medication. Liposome curcumin can significantly reduce tendon adhesion. Cytokine PDGF-BB can promote the proliferation of fibroblasts and capillaries in peritendon tissue, and promote tendon healing. The combination of curcumin and PDGF-BB can reduce tendon adhesion and accelerate tendon healing.

\section{Compliance with Ethical Standards}

Ethical approval: This study was reviewed and approved by ethical board of yangzhou university

Informed consent: Informed consent was obtained from all individual participants included in the study.

\section{References}

1. Schiffman SC, Li Y, Martin RC. The association of manganese superoxide dismutase expression in barrett's esophageal progression with mnTBAP and curcumin oil therapy. The Journal of surgical research. 2012; 176: 535-541.

2. Kim B, Kim HS, Jung EJ, Lee JY, Tsang BK, Lim JM, et al. Curcumin induces ER stress-mediated apoptosis through selective generation of reactive oxygen species in cervical cancer cells. Molecular carcinogenesis. 2016, 55: 918-928.

3. Zhang W, Li X, Franchini MC, Xu K, Locatelli E, Martin RC, et al. Controlled release of curcumin from curcumin-loaded nanomicelles to preven peritendinous adhesion during achilles tendon healing in rats. International journal of Nanomedicine. 2016; 11: 2873-2881.

4. He H, Lu Y, Qi J, Zhu Q, Chen Z, Wu W. Adapting liposomes for oral drug delivery. Acta pharmaceutica Sinica B. 2019; 9: 36-48.

5. Li ZL, Peng SF, Chen X, Yq Z, Lq Z, Liu W, et al. Pluronics modified liposomes for curcumin encapsulation: Sustained release, stability and bioaccessibility. Food research international (Ottawa, Ont). 2018; 108: 246-253.

6. Kaur H, Patro I, Tikoo K, Sandhir R. Curcumin attenuates inflammatory response and cognitive deficits in experimental model of chronic epilepsy. Neurochemistry international. 2015; 89: 40-50.

7. Evrova O, Buschmann J. In vitro and in vivo effects of pdgf-bb delivery strategies on tendon healing: A review. European cells \& materials. 2017; 34: 15-39.

8. Kovacevic D, Gulotta LV, Ying L, Ehteshami JR, Deng XH, Rodeo SA RhPDGF-BB promotes early healing in a rat rotator cuff repair model. Clinical orthopaedics and related research. 2015; 473: 1644-1654.

9. Younesi M, Donmez BO, Islam A, Akkus O. Heparinized collagen sutures for sustained delivery of PDGF-BB: Delivery profile and effects on tendonderived cells in-vitro. Acta biomaterialia. 2016; 41: 100-109.

10. Docheva D, Müller SA, Majewski M, Evans $\mathrm{CH}$. Biologics for tendon repair Advanced drug delivery reviews. 2015; 84: 222-239.

11. Evrova O, Houska J, Welti M, Bonavoglia E, Calcagni M, Giovanoli P, et al. Bioactive, elastic, and biodegradable emulsion electrospun degrapol tube delivering PDGF-BB for tendon rupture repair. Macromolecular bioscience. 2016; 16: 1048-1063. 